\title{
Interview
}

\section{Hypertensive Vasculopathy}

\section{An Exclusive Interview with Dr. Rhian M. Touyz, President of the International Society of Hypertension}

\author{
Jeong Bae Park
}

Department of Medicine/Cardiology, Cheil General Hospital, Kwandong University College

of Medicine, Seoul, South Korea

\begin{abstract}
An exclusive interview by Prof. Jeong Bae Park conducted with Dr. Rhian M. Touyz in Seoul while she was visiting for the Korean Society of Hypertension, May 10, 2014. In this interview, Dr. Touyz explains and describes hypertensive vasculopathy.

(C) 2014 S. Karger AG, Basel
\end{abstract}

\section{Curriculum Vitae}

Dr. Prof. Rhian Touyz, MB BCh, MSc (Med), PhD, FRCP, FRSE, is the director of the Institute of Cardiovascular and Medical Sciences, and BHF Chair of Cardiovascular Medicine at the University of Glasgow, Glasgow, UK. She is also the director of the BHF Excellence Award. Prof. Rhian Touyz is a clinician-scientist who focuses on hypertension research. She was the Canada Research Chair in Hypertension and was Professor of Medicine at the Kidney Research Centre, Ottawa Hospital Research Institute (OHRI)/University of Ottawa, Ottawa, Ont., Canada until 2011, when she moved to the Institute of Cardiovascular and Medical Sciences. Dr. Touyz received her BSc (Hons) in 1980, her MB BCh in 1984, her MSc in 1986 and her PhD in 1992 from the University of the

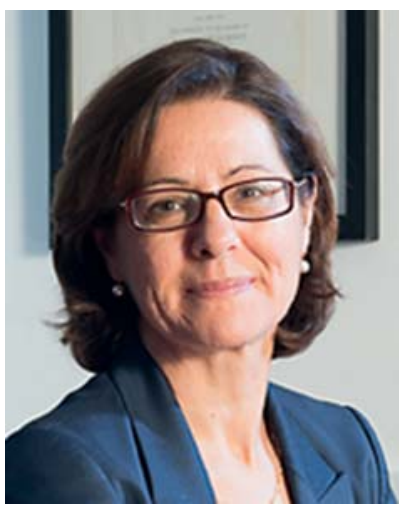

Dr. Prof. Rhian Touyz Witwatersrand, Johannesburg, South Africa. She completed a postdoctoral fellowship from 1992 to 1996 at the Clinical Research Institute of Montreal, Que., Canada. She has received numerous awards, including the Dahl Lecture Award of the American Heart Association (AHA) in 2005, the Robert M. 
Berne Distinguished Lecturer of the American Physiological Society in 2012 and the RD Wright Lecture Award of the High Blood Pressure Research Council of Australia. Dr. Touyz co-chaired the Recommendations Task Force of the Canadian Hypertension Education Program (CHEP), which is responsible for clinical hypertension guidelines. She is the president of the International Society of Hypertension and the past president of the Canadian Hypertension Society. She is the immediate past chair of the High Blood Pressure Research Council of the AHA. She is the Editor-in-Chief of Clinical Science, the Deputy Editor of Hypertension (AHA) and an Associate Editor of Pharmacological Reviews. She has trained many MSc and PhD students and has mentored over 30 postdoctoral fellows. Dr. Touyz has published over 325 original papers and reviews. Her areas of study include clinical and experimental hypertension, signal transduction, oxidative stress, ion transport, cell biology, vascular biology, adipose tissue biology and diabetes. She has a particular interest in translational research.

\section{Interview}

J.B.P.: Hypertensive vasculopathy seems like a vague terminology. It is not so clear how this term is different from other similar names, such as hypertensive vascular disorder. What actually is hypertensive vasculopathy in the context of hypertension?

R.M.T.: In its broadest sense, the term vasculopathy refers to the pathological changes that occur in vessels. In hypertension, functional and structural changes of small resistance arteries, such as endothelial dysfunction, increased contractility, vascular remodeling, impaired mechanics and vascular inflammation, characterize the changes that occur in hypertension and as such this phenotype is termed the vasculopathy of hypertension.

J.B.P.: How do vessels respond to changes after prolonged exposure to high blood pressure? How are these responses shown in different cell layers (endothelium, media and adventitia) and in small and large arteries?

R.M.T.: The lumen of resistance arteries is a function of the degree of vasodilation, regulated by endothelium-induced relaxation, and vasoconstriction, regulated by vascular smooth muscle cell (VSMC) contractility and by the structural characteristics of the vessel. Vasomotor control (relaxation/contraction) is responsible for the acute and rapid adaptation of the lumen diameter and may involve the activation of the sympathetic nervous system, the increased generation of nitric oxide or the release of vasoactive agents. Alterations in structural properties of the vascular wall constitute a dynamic process occurring in response to long-term hemodynamic modifications. Initially, structural changes are adaptive, but over time they become maladaptive resulting in abnormalities in vascular wall thickness and lumen diameter.

J.B.P.: Is vasculopathy of hypertension clinically relevant or simply a research interest?

R.M.T.: Both experimental and clinical evidence, using direct and indirect forms of assessment, indicate that small arteries in hypertension undergo characteristic changes including endothelial dysfunction and structural remodeling (vasculopathy). Importantly, treatment of hypertension and blood pressure lowering are associated with improved endothelial function and regression of remodeling. These findings indicate that the vasculopathy of hypertension is reversible. Whether blood pressure lowering itself normalizes vascular function and structure or whether improved vascular health leads to lower blood pressure remains unclear, as it is very difficult to establish what comes first, high blood pressure or the vasculopathy. However, the more we understand the molecular and cellular mechanisms of vascular injury in hypertension, the better prepared we will be to develop new therapies that target specific processes in the vasculature to prevent injury and vasculopathy and hopefully reduce blood pressure. On the other hand, beyond the research interests, treatment ap- 
Park et al.: Hypertensive Vasculopathy

proaches to promote vascular health, such as smoking cessation and drugs that have a direct vascular effect (calcium channel blockers, ARBs, etc.), have a global cardiovascular benefit and as such physicians should think about 'protecting' the vasculature when managing patients with hypertension. In summary, hypertensive vasculopathy is important academically and in the clinic.

J.B.P.: Regarding targeted therapy in hypertension, how is it possible to change the focus of treatment from reducing high blood pressure to treating hypertensive vasculopathy?

R.M.T.: The cornerstone of treatment is to protect the vasculature against damage and to maintain global vascular health. There are many 'lifestyle modifications' and nondrug strategies that should be promoted, such as smoking cessation, low fat diet and exercise. From a drug-target point of view, drugs that act on the vasculature can have direct vascular effects while still reducing blood pressure systemically. It is very difficult to dissociate direct vascular effects from blood pressure effects of drugs, and probably both factors are important in overall blood pressure reduction.

J.B.P.: It is known that the pharmacological inhibition of Rho kinase with fasudil or Y27632 suppresses the pressor response of Ang II but does not reduce blood pressure. Does this mean that it can be used as an antihypertensive drug for vascular protection without reducing blood pressure? Do you think it can be used in human hypertension?

R.M.T.: Human studies have demonstrated beneficial effects of Rho kinase inhibitors. Fasudil has been used clinically since the 1990s and effectively prevents vasospasm associated with subarachnoid hemorrhage, acute ischemic stroke, coronary artery spasm, pulmonary arterial hypertension, atherosclerosis, and in the regulation of vascular tone in hypertensive renal transplant recipients. Therapeutic use of Rho kinase inhibitors in human essential hypertension has not yet been approved but may be a potentially effective therapeutic strategy.

J.B.P.: Arterial calcification is one of the strong risk factors even in apparently healthy subjects. This is particularly true in coronary artery calcification. However, in clinical studies conducted in humans, statin therapy failed to show a reduction in calcification. Do you think that antihypertensive therapy with a renin angiotensin system blocker could prevent or reduce vascular calcification and in turn reduce cardiovascular events? Some animal studies have shown evidence for it but I am not sure if the same idea can be applied to humans.

R.M.T.: Vascular calcification is associated with the mineralization of the internal elastic lamina and elastic fibers within the media resulting in stiffened vessels and increased pulse pressure. Arterial calcification is not uncommon in aging, chronic kidney disease, diabetes, atherosclerosis as well as hypertension and is related to cardiovascular morbidity and mortality. Vascular calcification is a tightly controlled process similar to bone formation. VSMCs play a critical role in this process through their capacity to undergo phenotypic differentiation. Factors that trigger and promote VSMC osteogenic induction include abnormalities in mineral metabolism, particularly hyperphosphatemia and hypercalcemia. $\mathrm{AT}_{1} \mathrm{R}$ blockade can inhibit arterial calcification by disrupting vascular osteogenesis, suggesting that patients with vascular calcification may benefit therapeutically from Ang II receptor blockers, independently of the blood pressure-lowering effects. Although there is extensive experimental data showing that agents that interrupt the renin angiotensin system may improve vascular calcification, clinical studies are still lacking.

J.B.P.: It is commonly believed that there are no differences between men and women in the treatment of hypertension. Does this idea also apply to hypertensive vasculopathy?

R.M.T.: Experimental and clinical data indicate that sex has an important influence on vascular (patho)physiology, with women having more severe or different forms of vasculopathy than men, especially in the postmenopausal period. Risk conditions unique to women unrelated to pregnancy but linked to hypertension include polycystic ovarian syndrome, 
Park et al.: Hypertensive Vasculopathy

hypoestrogenemia of central origin and estrogen replacement. In addition, women have higher frequencies of vasculitis (lupus vasculitis, rheumatoid vasculitis, Raynaud's phenomenon, migraine, etc.). Functional alterations, such as changes in endothelial dysfunction, impaired vasodilator responses, increased vascular reactivity and remodeling (media thickening, atherosclerosis and inflammation), are more severe in (postmenopausal) women than men. This has been attributed, in part, to the fact that in women, vessels are continuously exposed to variable hormone levels, which may impact on vessel 'programming' for more severe functional alterations compared with men. Variations in plasma sex hormone levels and altered expression of estrogen, progesterone and testosterone receptors on endothelial cells and VSMCs may contribute to heterogeneity among women and gender differences in hypertensive vasculopathy.

In addition, reduced bone marrow-derived endothelial progenitor cells may contribute to impaired vascular repair in women with cardiovascular risks, including hypertension. Exaggerated vascular injury in women, especially in the postmenopausal period, may explain, in part, why hypertension and associated cardiovascular risk increase with aging in women. Gender differences in vascular oxidative stress have been implicated to play an important role in hypertensive vasculopathy associated with the sex hormone-related vasculopathy of hypertension.

J.B.P.: Thank you for a wonderful interview.

\section{Further Reading}

Briet M, Schiffrin EL: Treatment of arterial remodeling in essential hypertension. Curr Hypertens Rep 2013;15:3-9.

- Burger D, Touyz RM: Cellular biomarkers of endothelial health: microparticles, endothelial progenitor cells, and circulating endothelial cells. J Am Soc Hypertens 2012;6:85-99.

Montezano AC, Touyz RM: Molecular mechanisms of hypertension - reactive oxygen species and antioxidants: a basic science update for the clinician. Can J Cardiol 2012;28:288-295.

Montezano AC, Touyz RM: Reactive oxygen species, vascular Noxs, and hypertension: focus on translational and clinical research. Antioxid Redox Signal 2014;20:164-182.

Rizzoni D, Agabiti-Rosei E: Structural abnormalities of small resistance arteries in essential hypertension. Intern Emerg Med 2012;7:205-212.

-Savoia C, Burger D, Nishigaki N, Montezano A, Touyz RM: Angiotensin II and the vascular phenotype in hypertension. Expert Rev Mol Med 2011;13:e11.

Touyz RM, Briones AM: Reactive oxygen species and vascular biology: implications in human hypertension. Hypertens Res 2011;34:5-14. 